\title{
Preface to the proceedings of the 20th ILAS meeting, Leuven, Belgium, 2016
}

\author{
D. Farenick, B. Lemmens, M. Van Barel, and R. Vandebril
}

August 16, 2017

This special issue of Linear Algebra and its Applications contains the proceedings of the 20th meeting of the International Linear Algebra Society. The conference was hosted by the KU Leuven, University of Leuven, Belgium and took place from the 11th to the 15th of July, 2016.

The meeting was the largest one held so far and succeeded in bringing together 440 researchers from more than 45 countries over the world. We were in for a stimulating and research crossfertilizing week. Roughly 390 research lectures were presented including plenaries, lectures in mini-symposia, and contributed talks. The conference offered 10,000 minutes of mathematical knowledge expositions!

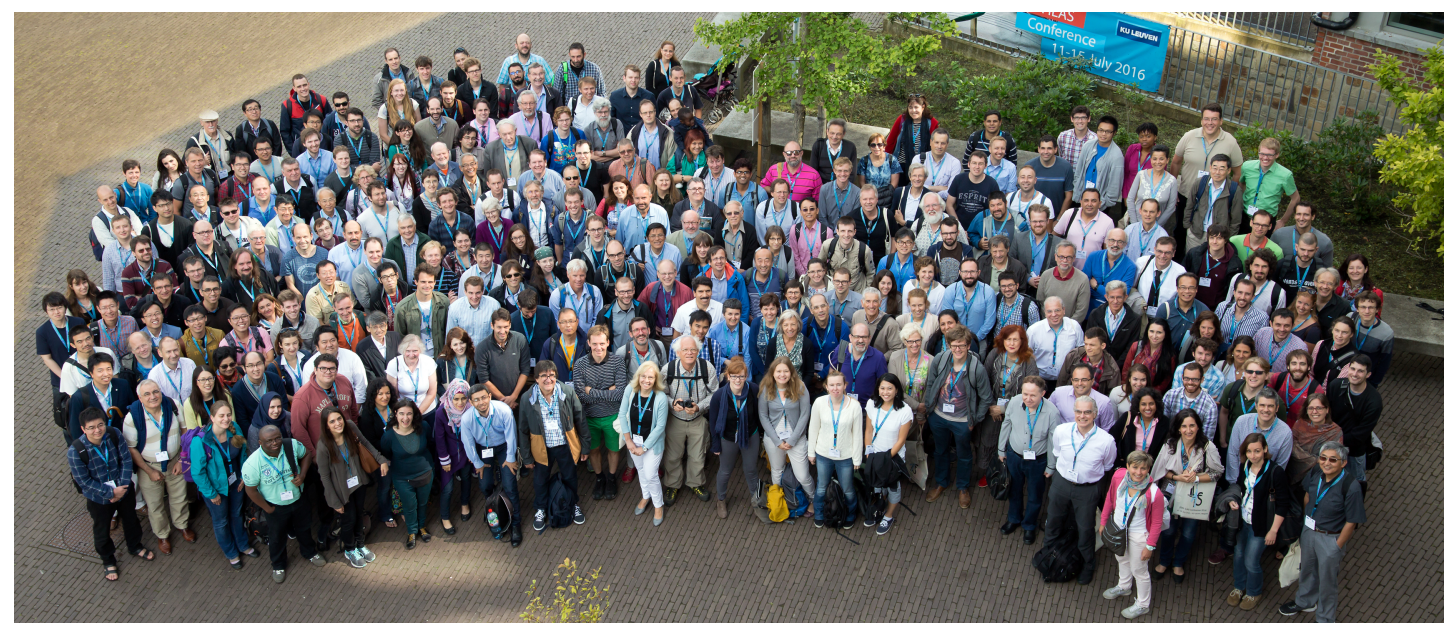

(Conference Foto, taken by Rob Stevens, KU Leuven)

The Local Organizing Committee consisted of Pierre-Antoine Absil, Thomas Mach, Karl Meerbergen, Wim Michiels, Leonardo Robol, Raf Vandebril, Wim Vanroose, and Marc Van Barel.

The Scientific Organizing Committee did a great job in carefully selecting the plenaries and minisymposia. The presented plenaries, ordered as the talks were presented, were Pablo A. Parrilo (LAA speaker), "Convex sets, matrix factorizations and positive semidefinite rank"; André Ran, "Eigenvalue perturbation theory of classes of structured matrices under generic structured small rank perturbations"; Monique Laurent, "On completely positive semidefinite matrices and their applications"; Lajos Molnar (LAMA speaker), "Preservers on positive matrices and operators"; Stefano Serra Capizzano, "Spectral Analysis and Fast Solvers for Hidden Structures"; Paul Van Dooren, "Polynomial Matrices and Dual Minimal Bases"; Fernando De Terán, "Solution of Sylvester-like equations and systems: Consistency, Uniqueness, and some applications"; Lieven De Lathauwer, "Between linear and nonlinear: an introduction to tensor methods"; Elizabeth S. Meckes, "Random orthogonal and unitary matrices".

The invited Minisymposia ranged from pure theoretical linear algebra to practical application oriented studies. The nine invited Minisymposia were: Data-Driven Model Reduction by Athanasios Antoulas; Matrix Equations by Peter Benner and Beatrice Meini; Tropical Algebra 
in Numerical Linear Algebra by James Hook, Jennifer Pestana, and Françoise Tisseur; Matrix Inequalities and Operator Means by Jean-Christophe Bourin and Takeaki Yamazaki; Linear Algebra and Quantum Computation by Chi-Kwong Li, Raymond Nung-Sing Sze, and Yiu Tung Poon; Multivariate Polynomial Computations and Polynomial Systems by Bernard Mourrain, Vanni Noferini, and Marc Van Barel; Image Restoration and Reconstruction by Marco Donatelli and James G. Nagy; Matrix Methods in Network Analysis by Dario Fasino and Francesco Tudisco; and Low Rank Tensor Approximation by André Uschmajew and Bart Vandereycken.

We would like to thank Scientific Organizing Committee for their work: Dario Bini (University of Pisa, Italy), Froilán Dopico (Universidad Carlos III de Madrid, Spain), Douglas Farenick (University of Regina, Canada), Heike Fassbender (Technische Universität Braunschweig, Germany), Fumio Hiai (Tohoku University, Japan), Steve Kirkland (University of Manitoba, Canada), Wim Michiels (KU Leuven, Belgium), Peter Semrl (University of Ljubljana, Slovenia), Françoise Tisseur (The University of Manchester, UK), Raf Vandebril (KU Leuven, Belgium), and Hugo Woerdeman (Drexel University, United States).

The Hans Schneider prize was offered to Paul Van Dooren at the banquet for his valuable contributions to the linear algebra society over the years. Paul Van Dooren received the Prize from Richard Brualdi and Peter Šemrl.

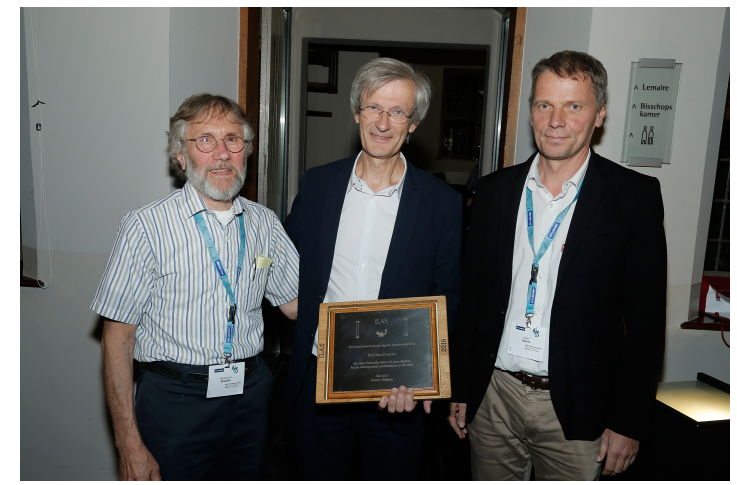

(Hans Schneider Award, foto taken by Rob Stevens, KU Leuven)

These proceedings have undergone the classical reviewing process to ensure the high standards of Linear Algebra and its Applications. The articles accepted illustrate the wide variety of topics discussed at the meeting. To conclude we would like to thank the authors for contributing to this journal issue, and also the referees whose valuable time was invested in reading all articles and offering suggestions to improve the quality of the presented articles. 